\title{
Réexamen de la justification du tritium dans l'industrie horlogère
}

\author{
CH. MURITH ${ }^{1}$, W. ZELLER ${ }^{1}$, M. HAMMANS ${ }^{2}$, A. ZELLER ${ }^{3}$
}

(Manuscrit reçu le 28 septembre 2005, accepté le $1^{\text {er }}$ février 2006)

RÉSUMÉ L'utilisation du tritium dans l'industrie horlogère pour amplifier la luminosité de la peinture luminescente peut être à l'origine de doses non négligeables au personnel de posage et conduit à la production de déchets radioactifs qui doivent être éliminés de façon correcte. Comme l'indiquent certaines enquêtes, bien des montres commercialisées n'ont pas la luminosité qu'est en droit d'attendre l'acheteur conformément à la norme concernant la radioluminescence pour les instruments horaires. Par ailleurs, il existe aujourd'hui des produits de substitution non radioactifs sur le marché. Enfin, la difficulté de maîtriser les héritages radiologiques provenant de l'industrie horlogère favorise inévitablement leur dissémination. L'ensemble de ces éléments est à la base du réexamen de la justification des peintures luminescentes radioactives. Les résultats exposés dans cet article confirment que la stratégie visant à renoncer naturellement à leur utilisation et à assister les entreprises dans le ramassage et l'élimination contrôlée des déchets est profitable à la radioprotection.

ABSTRACT Re-examination of the justification of tritium in the watch industry.

The use of tritium in the watch industry to amplify the luminosity of luminous paintings can induce significant doses to the working personal and also products radioactive wastes that have to be correctly eliminated. On one hand, as demonstrated by several investigations, a lot of commercialised watches do not have the required luminosity according to the ISO standard about radioluminescence for time measurement instruments. In the other hand substitution products without radioactivity are today available on the market. Finally, the difficulty to manage radioactive legacies from the watch industry inevitably contributes to disseminate them. All these considerations motivated the reexamination of the justification of luminous radioactive paintings. Results and approaches exposed in this paper confirm that the strategy to naturally abandon the use of luminous radioactive paintings and to assist the producers in collecting and in eliminating in a controlled manner the resulting radioactive wastes is beneficial to radiation protection.

Keywords: H-3 / Ra-226 / luminous painting / radiation protection / watch industry

\section{Introduction}

Depuis 1993, l'Institut de radiophysique appliquée (IRA, 1993) à Lausanne est mandaté par l'OFSP ${ }^{1}$ pour effectuer des mesures de radioactivité dans les montres.

\footnotetext{
Office fédéral de la santé publique, Radioprotection, 3003 Bern, Suisse.

Caisse nationale suisse d'assurance (Suva), 6002 Lucerne, Suisse.

3 Entreprise RC-Tritec, BP 147, 9053 Teufen, Suisse.
} 
TABLEAU I

Justification de l'utilisation du tritium en regard de la norme ISO 3157. Justification of tritium use according to ISO 3157 standard.

\begin{tabular}{cccccccc}
\hline Montre & $\begin{array}{c}\text { Activité } \\
{[\mathrm{MBq}]}\end{array}$ & $\begin{array}{c}\text { Intensité } \\
{[\mathrm{ncd}]}\end{array}$ & justifié & Montre & $\begin{array}{c}\text { Activité } \\
{[\mathrm{MBq}]}\end{array}$ & $\begin{array}{c}\text { Intensité } \\
{[\mathrm{ncd}]}\end{array}$ & justifié \\
\hline 1 & 55,6 & 161,2 & oui & 12 & 28 & 100,7 & oui \\
2 & 20,5 & 58,7 & oui & 13 & 36,3 & 98,8 & oui \\
3 & 65,8 & 117,4 & oui & 14 & 68 & 348,6 & oui \\
4 & 0,4 & 0,29 & non & 15 & 2,49 & 3,1 & non \\
\hline 5 & 16,3 & 22,8 & non & 16 & 21,4 & 57,2 & oui \\
6 & 7,16 & 10,4 & non & 17 & 0,2 & 18,8 & non \\
\hline 7 & 2,24 & 7,74 & non & 18 & 3,2 & 10,2 & non \\
8 & 27,8 & 36,8 & non & 19 & 17,9 & 44,1 & oui \\
9 & 10,6 & 20,4 & non & 20 & 46,3 & 119,1 & oui \\
10 & 19 & 29,1 & non & 21 & 85,9 & 226,4 & oui \\
11 & $<0,001$ & 1,2 & non & 22 & 10,0 & 34,2 & non \\
\hline
\end{tabular}

Le tableau I illustre les résultats qui remettent partiellement en question la justification de l'utilisation du tritium en regard de la norme ISO 3157 (ISO, 1991) concernant la radioluminescence pour les instruments horaires :

$$
\begin{gathered}
\text { activité }{ }^{3} \mathrm{H}<277 \text { mega-becquerel (MBq) et intensité lumineuse } \\
>45 \text { nano-candela (ncd). }
\end{gathered}
$$

En effet, sur 22 montres analysées 12 ne satisfont pas à cette norme. Le tritium a une demi-vie de 12 ans. Si au bout de 12 ans l'activité de tritium a diminué de moitié, pour la peinture luminescente la période effective se situe entre 3 et 5 ans, car une part du tritium est diffusée hors de la montre (Fig. 1). Par exemple une montre de $100 \mathrm{ncd}$ n'aura plus que la luminosité minimale requise de $45 \mathrm{ncd}$ au bout de 5 ans.

Suite à la concertation entre l'Office fédéral de la santé publique (OFSP), la Fédération de l'industrie horlogère suisse (FH), et la Caisse nationale d'assurance (Suva), les entreprises concernées ont pris conscience du problème et ont décidé d'abandonner certaines lignes de produits dès l'épuisement des stocks et de remplacer le tritium par un produit alternatif dans toute la collection. Parmi ces alternatives les pigments luminescents Super-Luminova, qui fonctionnent à la manière d'une batterie de lumière permettent d'améliorer la capacité de stockage de luminosité. Après une exposition suffisante au soleil ou à la lumière artificielle, l'énergie lumineuse est restituée de nuit durant des heures. Le processus d'activation et de phosphorescence peut se répéter quasi infiniment sans la 


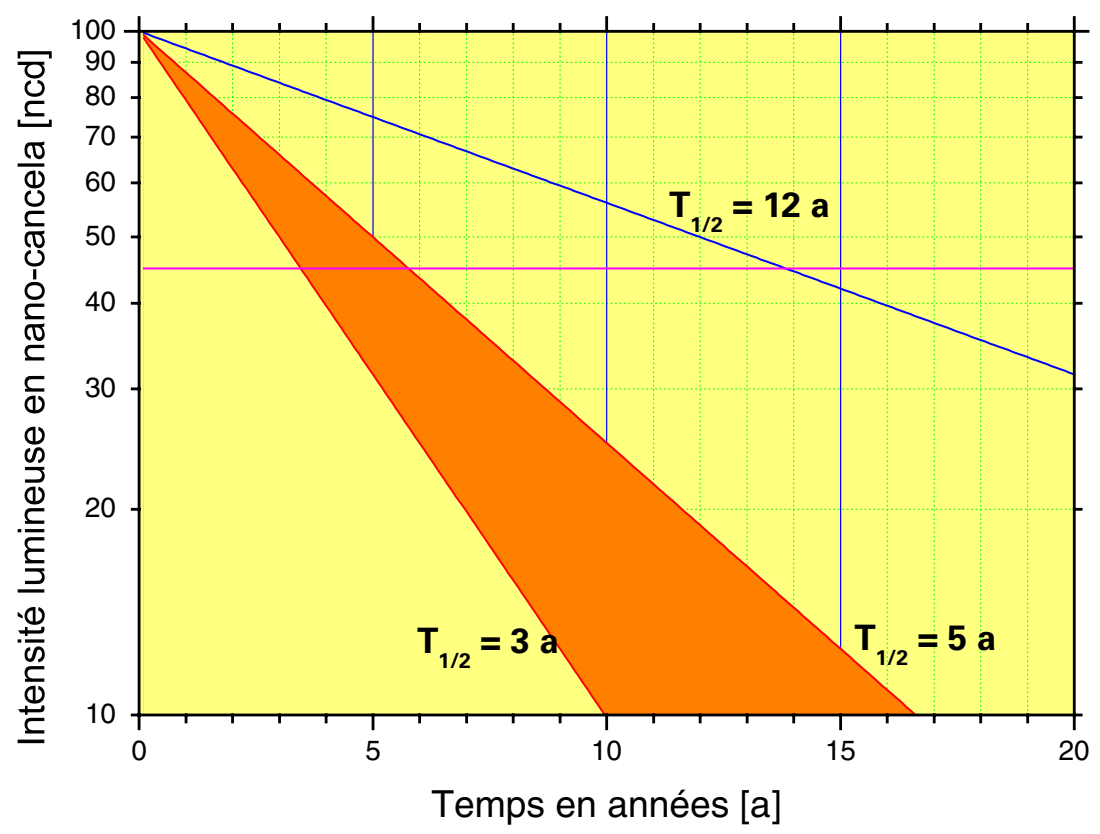

Figure 1 - Décroissance de la luminosité dans les montres tritium.

Decrease of luminosity in tritium watches.

moindre défaillance. Les pigments en question sont constitués d'une substance phosphorescente pure à base d'aluminate alcalinoterreux et ne comportent aucune trace de substance radioactive. Plus d'informations sont disponibles auprès de la société RC-Tritec (Teufen, www.rctritec.com).

\section{Réglementation suisse}

La réglementation suisse prévoit spécifiquement le cas des montres avec peinture luminescente (article 125c) de l'ordonnance sur la radioprotection (ORaP, 1994) et exempte d'autorisation la distribution, l'importation, l'exportation et l'utilisation de montres conformes aux normes ISO 3157 et 4168 . Les objets peints constituent des sources non scellées (5 à $10 \%$ de l'activité contenue dans la peinture est diffusée par an). En matière d'objets contenant des ampoules de tritium gazeux qui constituent des sources scellées, certains (montres, matériel militaire, ...) font l'objet d'une homologation (autorisation générique de détention, articles 128 à 130 de l'ORaP) par l'OFSP. L'homologation prévoit la 
TABLEAU II

Limites annuelles pour les rejets des entreprises Tritec et Microtec. Annual limits concerning the releases of Tritec and Microtec companies.

\begin{tabular}{lccc}
\hline Rejets & liquides & \multicolumn{2}{c}{ atmosphériques } \\
& $\mathrm{H}-3$ & $\mathrm{H}-3(\mathrm{HT})$ & $\mathrm{H}-3(\mathrm{HTO})$ \\
\hline RC-Tritec, Teufen & $20 \mathrm{GBq}$ & $15 \mathrm{TBq}$ & $20 \mathrm{TBq}$ \\
Mb-microtec, Niederwangen & $40 \mathrm{GBq}$ & $370 \mathrm{TBq}$ & $37 \mathrm{TBq}$ \\
\hline
\end{tabular}

reprise par le fournisseur des montres en fin d'utilisation si leur détenteur le souhaite.

\section{3. Évolution des émissions des entreprises de l'industrie horlogère en Suisse}

En matière de rejets, les limites annuelles fixées pour les entreprises de production du secteur horloger sont reportées dans le tableau II. Ces entreprises sont tenues de communiquer à l'autorité de surveillance, la Suva, le bilan de leurs émissions (Figs. 2 et 3). Celles-ci ont atteint 10 à 30 pour cent des valeurs limites, jusqu' aux années 90. Dans la figure 2, la différence entre les deux courbes concernant les rejets atmosphériques est due au tritium gazeux utilisé pour la fabrication des betalight (societé MB-Microtec, Niederwangen, www.mbmicrotec.com).

Dans le voisinage de ces entreprises, le tritium est analysé dans les précipitations, l'humidité de l'air et les eaux superficielles. Pour ce qui est des ateliers de La Chaux-de-Fonds spécialisés dans l'application de peintures luminescentes, le tritium est également analysé dans les eaux de lavage des fumées de l'usine d'incinération et dans les eaux usées de la station d'épuration locale (STEP). Les décharges sont surveillées de manière ciblée par l'analyse des eaux d'infiltration. Comme on peut le voir sur la figure 4, l'utilisation de tritium par l'industrie horlogère a diminué approximativement d'un facteur 100 au cours de ces dix dernières années, du fait que plusieurs horlogers ont renoncé au tritium. L'évolution de ces bilans est publiée annuellement par l'OFSP (2004a) et montre une diminution correspondante des concentrations de tritium dans le Doubs.

\section{4. Évolution des doses affectant le personnel de l'industrie horlogère}

En tant que personnes professionnellement exposées aux rayonnements, le personnel de l'industrie horlogère est tenu d'être formé en radioprotection et est 


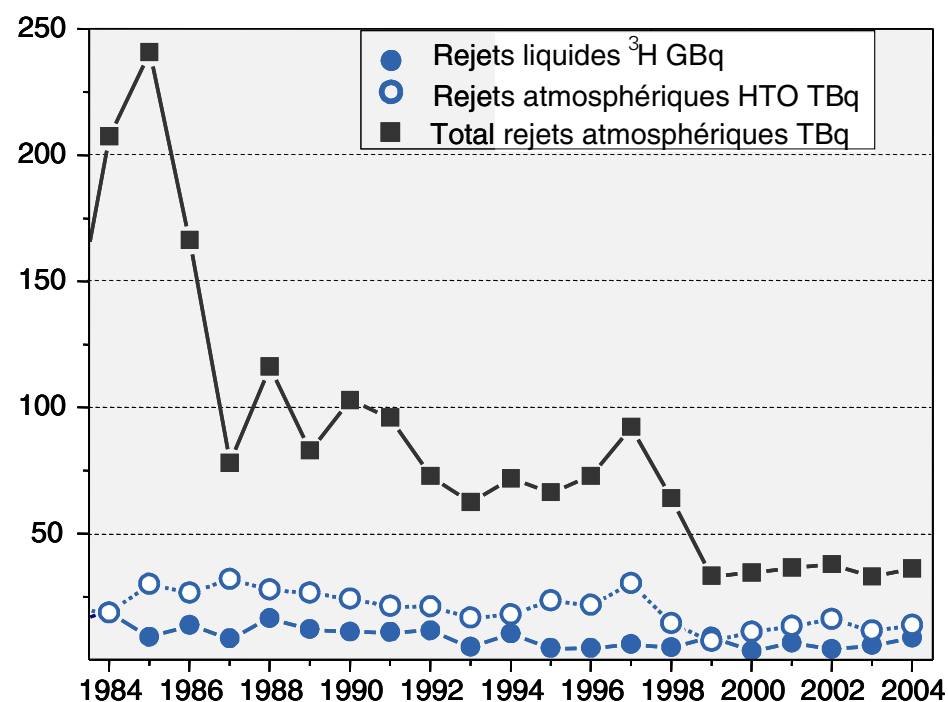

Figure 2 - Émissions de tritium de l'entreprise MB-Microtec AG (Niederwangen/BE) par l'air vicié et les eaux usées.

Tritium atmospheric and liquid emissions of MB-Microtec (Niederwangen/BE).

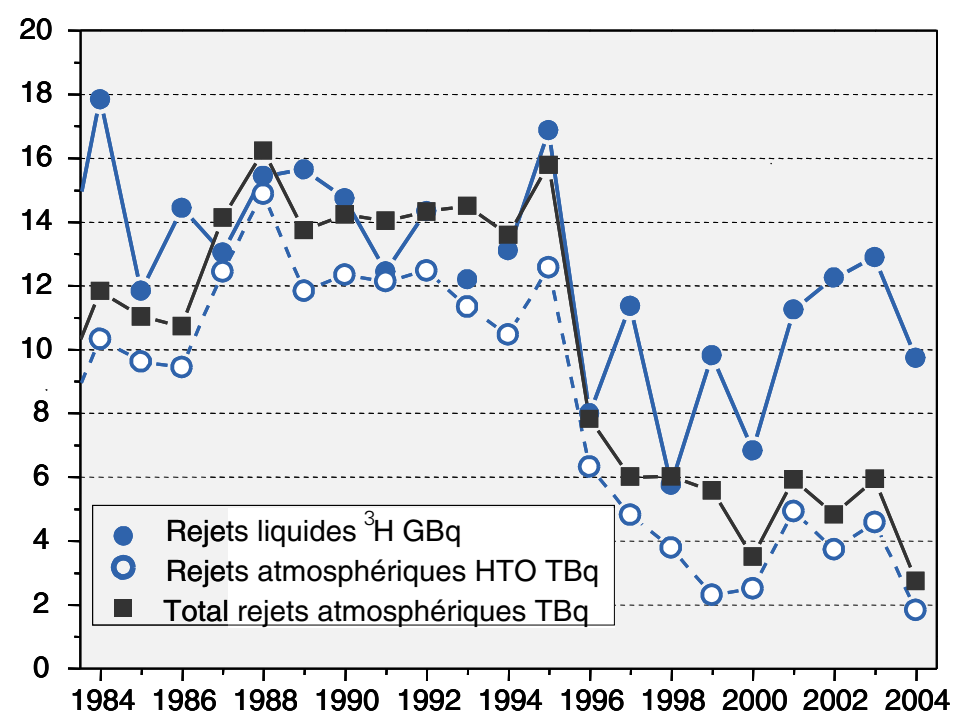

Figure 3 - Émissions de tritium de l'entreprise RC-Tritec AG (Teufen/AR) par l'air vicié et les eaux usées. Tritium atmospheric and liquid emissions of RC-Tritec (Teufen/AR). 


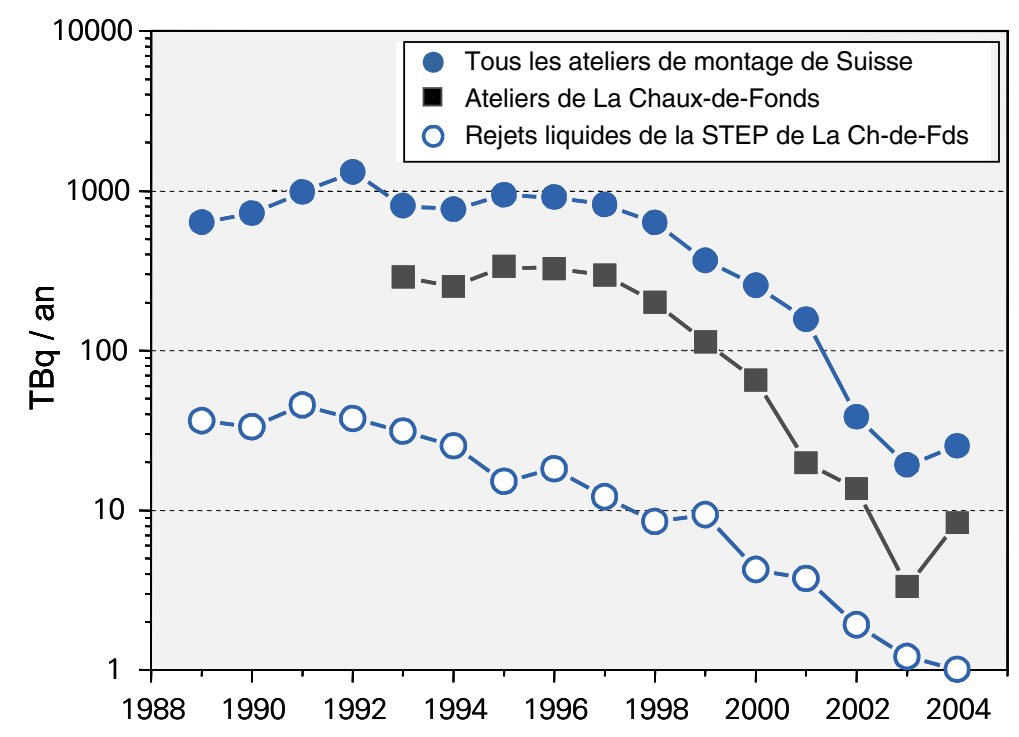

Figure 4 - Utilisation de tritium dans l'industrie horlogère en Suisse, à La Chaux-de-Fonds et quantité de tritium dans l'écoulement de la STEP de La Chaux-de-Fonds.

Tritium use in the Swiss watch industry, in La Chaux-de-Fonds and tritium content in the water purification station (STEP) of La Chaux-de-Fonds.

soumis à un suivi dosimétrique et médical, comprenant en particulier un contrôle régulier de l'urine. La Suva, autorité de surveillance pour le secteur industriel a en outre fixé des buts plus contraignants aux entreprises que ne le prévoit la loi (20 mSv/an) en vertu du principe d'optimisation (article 6 de l'ORaP). Ainsi la dose cumulée sur une année en raison de l'entreposage d'aiguilles et de cadrans peints au tritium ne doit pas dépasser $1 \mathrm{mSv}$. Pour l'air respiré (40 h par semaine et 50 semaines par an) une concentration de $25 \mathrm{kBq} / \mathrm{m}^{3}$ implique une dose de 1 $\mathrm{mSv} / \mathrm{an}$. Pour la concentration moyenne de tritium dans l'urine, $54 \mathrm{kBq} / \mathrm{L}$ conduit à cette dose. Avant 1995, la dose collective par irradiation externe et interne du personnel de l'industrie horlogère était comparable à celle du personnel d'une centrale nucléaire ( 910 personnes-mSv pour 363 personnes essentiellement des jeunes femmes pour les entreprises de peintures luminescentes et 920 personnesmSv pour les 783 employés surtout des hommes d'âge moyen pour la centrale nucléaire de Gösgen). En ce qui concerne la dose interne, le $95 \%$ de la dose collective par incorporation était imputable aux activités de l'industrie horlogère et touchait principalement de jeunes femmes, le $5 \%$ restant provenant d'autres secteurs. Depuis, l'action des industriels encouragée par l'OFSP sans pression 


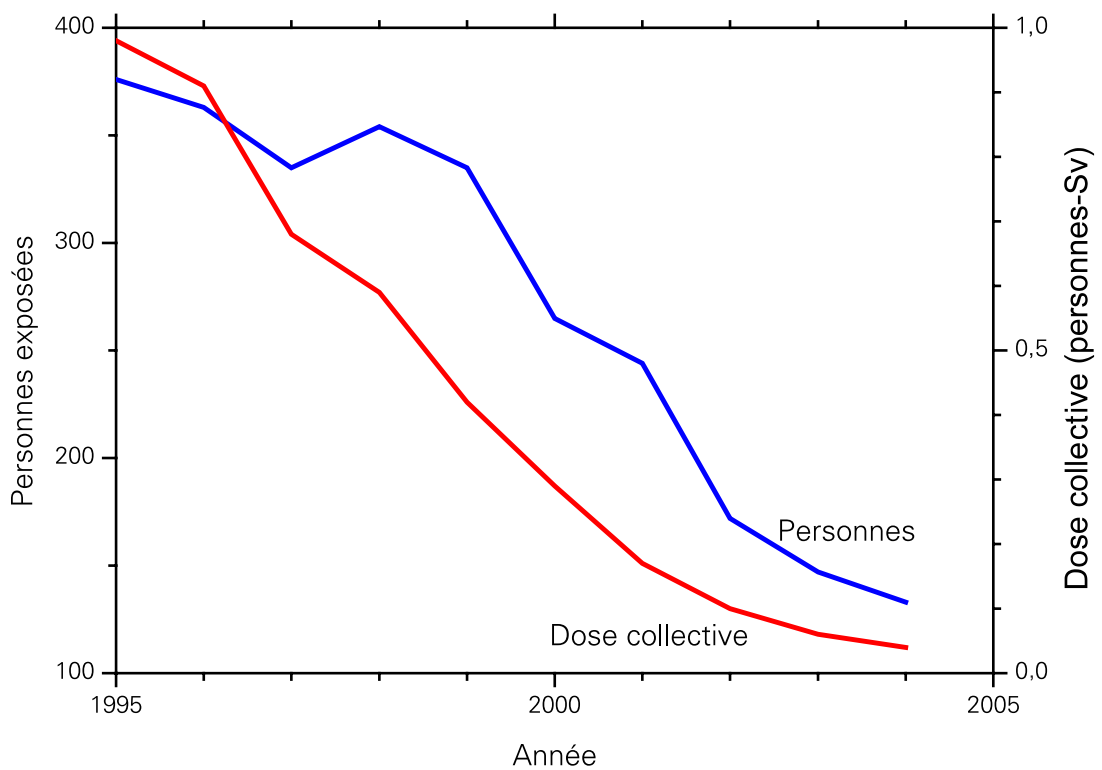

Figure 5 - Évolution de la dose collective et des personnes exposées dans l'industrie horlogère. Evolution of collective dose and exposed persons in the Swiss watch industry.

réglementaire formelle a porté ses fruits ; comme le montre la figure 5, on constate une nette diminution de la dose collective qui résulte d'une part de l'optimisation des processus de travail dans les entreprises utilisant des couleurs luminescentes et d'autre part du renoncement progressif à leur utilisation.

Les informations détaillées sur la dosimétrie individuelle sont publiées annuellement par l'OFSP (2004b) qui tient à jour le registre central des doses.

\section{Gestion des déchets}

La définition des déchets et les principes de leur gestion sont clairement explicités dans l'article 25 de la loi sur la radioprotection (LRaP, 1991) : «Par déchets radioactifs on entend les substances radioactives et les matières contaminées par elles qui ne seront pas réutilisées. Les déchets radioactifs ne provenant pas de Suisse ne peuvent être importés pour être éliminés que si notre pays s'est engagé par un accord de droit international public à les prendre en charge ».

Pour les montres un tel accord n'existe pas. En conséquence, même si la Suisse est à même techniquement d'éliminer de tels déchets, elle ne peut prendre en 
charge que ceux présents en Suisse et en aucun cas ceux qui proviennent de l'étranger.

En 1999, L'OFSP, la FH et la Suva se sont réunis pour discuter de l'avenir des substances radioactives dans l'industrie horlogère. Dans une action concertée et limitée dans le temps, il a été décidé d'assister les entreprises dans les tâches d'élimination des déchets radioactifs selon des conditions spéciales. Le but visé étant d'inciter les entreprises à prendre conscience de la problématique et à faire le pas vers l'introduction de produits alternatifs. L'augmentation des coûts dans l'élimination des déchets radioactifs et les préparatifs en vue de leur élimination représentent certainement le problème principal.

Des actions de ramassage ont été lancées depuis 1985. En 2004, l'action de ramassage de la Suva a par exemple permis de collecter 27000 montres avec cadrans au radium $\left(100 \mathrm{MBq}{ }^{226} \mathrm{Ra}\right)$ et 68000 montres avec cadrans au tritium (7 TBq). Ces déchets sont conditionnés par l'entreprise RC-Tritec avant d'être amenés au centre fédéral de ramassage de déchets radioactifs (PSI) pour leur entreposage.

Le contrôle des entreprises permet d'estimer qu'il reste une centaine de milliers de cadrans radioluminescents au tritium dans les stocks de rhabillage chez les fabricants. La préparation des déchets radioactifs et une éventuelle décontamination des lieux sont confiées à des spécialistes. Le contrôle sur place des emballages qui seront pris en charge par un transporteur détenteur des autorisations nécessaires est assumé par l'Institut Paul Scherrer (PSI). Le contrôle final de l'entreprise incombe à la Suva.

Pour les déchets de faible activité en tritium (quelques centaines de GBq équivalent à un millier de montres), une stratégie d'élimination raisonnable consiste à convaincre des usines d'incinération de faire une demande ponctuelle d'autorisation auprès de l'OFSP en vue de procéder à l'incinération contrôlée de ces déchets dans le respect des limites de la législation en vigueur. Cela représente plusieurs tonnes de matériel à incinérer. Ce processus a été utilisé avec succès à $\mathrm{La}$ Chaux-de-Fonds, comme l'ont confirmé les mesures dans l'environnement et dans l'eau de lavage des fumées effectuées par l'autorité de surveillance, responsable pour la radioactivité de l'environnement (OFSP, 2003).

Pour des déchets présentant des activités en tritium plus élevées, la procédure d'élimination passe par la solidification des déchets dans des fûts métalliques et leur transport au centre fédéral de ramassage des déchets radioactifs (PSI) pour leur entreposage avec examen du devenir de ces déchets après une période de 100 ans. 
Pour les montres en circulation, il semble illusoire d'entreprendre des mesures efficaces. Leur élimination sauvage dans les usines d'incinération implique essentiellement une augmentation du niveau de tritium dans l'eau de lavage de fumées.

Concernant les déchets au radium qui ne sont abordés ici que de manière marginale, la situation est plus délicate. Une incinération de matériaux contaminés (tables, revêtement de sol, ...) provenant des anciens ateliers nécessiterait une modification de la loi sans garantir que les usines d'incinération se déclarent prêtes à accepter des cendres de radium. Déjà d'un point de vue politique une telle solution s'avère complexe à défendre. Il convient ici de mentionner que les coûts liés au triage et à l'élimination correcte de quelques $\mathrm{kBq}$ de radium représentent des montants importants qui sont à la charge de l'État. Suite à l'assainissement des entreprises Radium-Chemie de Teufen et Merz \& Benteli de Berne (Rapport, 1982), la solution préconisée a par exemple consisté pour la première à entreposer sur place les déchets $\left(100 \mathrm{~m}^{3}\right.$ contenant $1 \mathrm{GBq}$ de radium) dont la localisation est définie dans le cadastre respectivement pour la seconde à transporter la terre et les débris de construction à la décharge de Teuftal. Pour les ateliers de posage de la région de La Chaux-de-Fonds, une démarche similaire (assainissement et entreposage local) apparaît actuellement la plus pertinente.

\section{Héritages en tritium de l'industrie horlogère : inventaire et gestion}

L'estimation des héritages en tritium, les producteurs et utilisateurs concernés ainsi que les aspects de radioprotection et les actions limitant la dissémination des produits au tritium sont résumés dans le tableau III.

TABLEAU III

Inventaire et gestion des héritages en tritium.

Inventory and management of tritium legacies.

\begin{tabular}{ll}
\hline Utilisation & $\sim 1963-2003:$ l'activité totale traitée peut être estimée à : \\
& 40 ans $\times 25 \mathrm{kgCi} /$ an soit $~ 40000 \mathrm{TBq}\left(10^{6} \mathrm{Ci}\right)$ tritium \\
Producteurs & $\begin{array}{l}\text { Merz \& Benteli, Berne }(1964-1980) \\
\text { Radium-Chemie, Teufen }(1962-2005)\end{array}$ \\
\multirow{2}{*}{ Radilisateurs } & $1970: \sim 25$ ateliers $; 1990: \sim 13 ;$ \\
& $2004:$ encore 5 autorisations $(<20 \mathrm{TBq})$ \\
Conséquencestion & $\begin{array}{l}\text { Depuis } 1963: \text { Inventaire et contrôle de tous les ateliers } \\
\text { Cours de formation et surveillance de tous les employés par contrôle mensuel dans l'urine, } \\
\text { autorité de surveillance (Suva) }\end{array}$ \\
& $\begin{array}{l}\text { Production : action de ramassage (décharges et usines d'incinération) } \\
\text { Cadrans + aiguilles : action de ramassage, fonderie, ferrailleur, décharges, lavage par solution } \\
\text { des usines d'incinération, } \\
\text { Montres non utilisées : usine d'incinération, décharges } \\
\text { Domaine militaire : (compas, viseur, instruments divers) ramassage }\end{array}$ \\
\hline
\end{tabular}


Parmi les formes des héritages en tritium, on compte :

- restes de peintures : action de ramassage,

- instruments de travail assez fortement contaminés : action de ramassage,

- cadrans, aiguilles et autres composants : action de ramassage,

- objets faiblement contaminés : mobilier, système d'aération, sol, habits, ...,

- les contaminations de l'environnement sont naturellement éliminées.

Il faut donc enlever le matériel contaminé dont le « dégazage » charge continûment l'air ambiant.

Les solutions pragmatiques d'élimination des héritages en tritium doivent respecter les aspects réglementaires suivants :

- incinérateur autorisé : (demande de l'usine et autorisation de l'OFSP),

- respect de l'ordonnance sur la propreté de l'air,

- incinération exclusive de ${ }^{3} \mathrm{H},{ }^{14} \mathrm{C},{ }^{35} \mathrm{~S}$,

- activité hebdomadaire $\max =1000 \times \mathrm{LA}:$ pour ${ }^{3} \mathrm{H}: 300 \mathrm{GBq}(8 \mathrm{Ci}) /$ semaine.

Les résidus de l'incinération et de lavage des fumées doivent être traités comme déchets (HTO). L'expérience suisse concernant l'élimination des héritages en tritium a mis en évidence la problématique et les aspects suivants.

La problématique: peu de stations d'incinération bénéficient d'une autorisation et lorsqu'elles en ont une elle est limitée (incinération exclusive de déchets d'entreprise du canton pour Cridor/NE et limitation des rejets HTO pour Zwilag/AG). Les coûts sont élevés (8 à $15 \mathrm{CHF} / \mathrm{kg}+1000 \mathrm{CHF}$ de taxe radioprotection). Le manque d'intérêt en faveur d'un centre national d'incinération et la réticence des autres stations de peur d'une publicité « incinération de déchets radioactifs », bien qu'il leur soit livré à toutes des déchets contenant du tritium, sont regrettables.

Les aspects radioprotection: lors de l'incinération la majeure partie de l'activité de ${ }^{3} \mathrm{H}$ est retenue sous forme HTO dans l'eau de lavage des fumées, $\sim 12 \%$ s'échappant par la cheminée. Il en résulte un bilan clair et aucun rejet non contrôlé dans l'environnement comme l'a confirmé l'expérience avec Cridor. Les incinérations contrôlées constituent un faible pourcentage des livraisons en regard des éliminations non contrôlées d'après les analyses des eaux de lavages des stations d'incinération. L'inventaire ${ }^{3} \mathrm{H}$ de l'industrie horlogère finit de toute manière dans le système aquatique. Par contre avec l'incinération, l'élimination se fait de manière contrôlée et diluée et le tritium n'atteint pas la nappe phréatique. 


\section{Conclusions}

L'utilisation de peintures luminescentes au tritium semble en voie de disparition à la suite des actions des industriels encouragées par l'OFSP et la Suva. Le seul procédé de radioluminescence au tritium est pratiquement l'utilisation d'ampoules au tritium gazeux.

Les lois du marché (baisse de la demande, interdiction d'importation dans certains pays) règlent d'elles-même la question du tritium. Certes pour la majorité des montres au tritium une personne qui en porte une en permanence n'accumulera jamais une dose effective supérieure à $10 \mu \mathrm{Sv}$ par an (pour des montres de plongée $<50 \mu$ Sv/an). La pression des autorités reste cependant justifiée pour accélérer la diminution des doses reçues par le personnel et pour un abandon définitif du ${ }^{3} \mathrm{H}$ car l'utilisateur du produit final n'en retire qu'un intérêt modéré et que des solutions alternatives existent. Pour les entreprises, l'abandon des peintures luminescentes a aussi des avantages. Ces dernières n'ont plus besoin d'autorisations pour la manipulation de substances radioactives, ni d'experts en radioprotection. De plus, la gestion des déchets radioactifs tombe et les problèmes liés à l'exportation de montres contenant du tritium deviennent caducs.

La gestion des déchets radioactifs provenant de l'industrie horlogère en Suisse se base sur des actions de ramassage, d'élimination contrôlée dans les usines d'incinération et de vigilance concernant le matériel militaire et les matériaux contaminés provenant des anciens ateliers de posage de peintures luminescentes. Ces mesures permettent sans nul doute d'éviter une dissémination sauvage de ces déchets radioactifs. La problématique des héritages radiologiques a été examinée par la Commission fédérale de protection contre les radiations et de surveillance de l'environnement (CPR, 2003). La CPR recommande les démarches suivantes pour favoriser l'élimination des déchets de l'industrie horlogère:

- élaboration de la possibilité d'éliminer les déchets à faible teneur de tritium à moindre coût pour éviter que rien ne soit fait ou que la décontamination et l'élimination n'interviennent de manière « sauvage »;

- plus l'élimination contrôlée est simple, plus rapidement et exhaustivement les matériaux contaminés avec du tritium disparaîtront de la circulation et ne représenteront plus de risque

- éviter les décharges et les incinérations sauvages,

- éviter le tourisme d'objets contenant du tritium ;

- élaboration de la possibilité légale d'incinérer des déchets solides et liquides à faible teneur de tritium sous des conditions raisonnables dans des usines d'incinération régionales ou des fours spéciaux (selon la procédure et la surveillance du modèle Cridor qui a fait ses preuves) ;

- examiner les solutions pertinentes dans le respect de la législation pour l'élimination des héritages radium (dilution). 


\section{RÉFÉRENCES}

CPR (2003) Recommandation de la CPR concernant la gestion des héritages radiologiques. http://www. ksr-cpr.admin.ch/pdf/f/Heritages_cpr.pdf

IRA (1993) Enquêtes sur la conformité des montres à la norme ISO 3157 effectuées par l'IRA, www.chuv.ch/public/instituts/ira/

ISO (1991) Norme ISO 3157, Radioluminescence pour les instruments horaires.

LRaP (1991) Loi sur la radioprotection du 22 mars 1991. http://www.admin.ch/ch/f/rs/8/814.50.fr.pdf

OFSP (2003) Elimination de déchets faiblement contaminés au tritium dans la station d'incinération d'ordures ménagères de La Chaux-de-Fonds (Chapitre 9.3). http://www.bag.admin.ch/strahlen/ pdf/archives/Radio_Rapport_2003_complet.pdf

OFSP (2004a) Rapport de division de l'OFSP. http://www.bag.admin.ch/strahlen/pdf/Jahresbericht2004_f.pdf

OFSP (2004b) Rapport de dosimétrie de l'OFSP. http://www.bag.admin.ch/strahlen/pdf/Dosimetrie2004.pdf

ORaP (1994) Ordonnance sur la radioprotection du 22 juin 1994. http://www.admin.ch/ch/f/rs/8/ 814.501.fr.pdf

Rapport (1982) Assainissement des entreprises RC-Tritec et Merz \& Benteli, 26 rapport de la commission fédérale de surveillance de la radioactivité, 1982. 